\title{
Altered expression of selected microRNAs in melanoma: Antiproliferative and proapoptotic activity of miRNA-155
}

\author{
LAURETTA LEVATI $^{1}$, ESTER ALVINO ${ }^{2}$, ELENA PAGANI ${ }^{1}$, DIEGO ARCELLI ${ }^{1}$, \\ PATRIZIA CAPORASO ${ }^{1}$, SERGIO BONDANZA ${ }^{3}$, GIANPIERO DI LEVA ${ }^{4}$, MANUELA FERRACIN ${ }^{5}$, \\ STEFANO VOLINIA $^{4,6}$, ENZO BONMASSAR ${ }^{2,7}$, CARLO MARIA CROCE ${ }^{4}$ and STEFANIA D'ATRI ${ }^{1}$ \\ ${ }^{1}$ Laboratory of Molecular Oncology, Istituto Dermopatico dell'Immacolata-IRCCS, Via dei Monti di Creta 104, \\ I-00167 Rome; ${ }^{2}$ Department of Medicine, Institute of Neurobiology and Molecular Medicine, National Council \\ of Research, Via Fosso del Cavaliere 100, I-00133 Rome; ${ }^{3}$ Laboratory of Tissue Engineering and Cutaneous \\ Physiopathology, Istituto Dermopatico dell'Immacolata-IRCCS, Via dei Monti di Creta 104, I-00167 Rome, Italy; \\ ${ }^{4}$ Department of Molecular Virology, Immunology and Medical Genetics and Comprehensive Cancer Center, \\ Ohio State University, 460 West 12th Avenue, Columbus, OH 43210, USA; ${ }^{5}$ Department of Experimental and \\ Diagnostic Medicine and Interdepartment Center for Cancer Research, University of Ferrara, Via Luigi Borsari 46; \\ ${ }^{6}$ DAMA, Data Mining for Microarray Analysis, Department of Morphology and Embryology, University of Ferrara, \\ Via Fossato di Mortara 64/b, I-44100 Ferrara; ${ }^{7}$ Department of Neuroscience, School of Medicine, \\ University of Rome 'Tor Vergata', Via Montpellier 1, I-00133 Rome, Italy
}

Received February 24, 2009; Accepted April 29, 2009

DOI: 10.3892/ijo_00000352

\begin{abstract}
Altered expression of microRNAs (miRNAs) has been detected in cancer, suggesting that these small non-coding RNAs can act as oncogenes or tumor suppressor genes. In the present study, we investigated the expression of miRNA-17-5p, miRNA-18a, miRNA-20a, miRNA-92a, miRNA-146a, miRNA-146b and miRNA-155 by real-time quantitative RT-PCR in a panel of melanocyte cultures and melanoma cell lines and explored the possible role of miRNA-155 in melanoma cell proliferation and survival. The analyzed miRNAs were selected on the basis of previous studies strongly supporting their involvement in cancer development and/or progression. We found that miRNA-17-5p, miRNA18a, miRNA-20a, and miRNA-92a were overexpressed, whereas miRNA-146a, miRNA-146b and miRNA-155 were down-regulated in the majority of melanoma cell lines with respect to melanocytes. Ectopic expression of miRNA-155 significantly inhibited proliferation in 12 of 13 melanoma cell lines with reduced levels of this miRNA and induced apoptosis in 4 out of 4 cell lines analyzed. In conclusion, our data further support the finding of altered miRNA expression in
\end{abstract}

Correspondence to: Dr Stefania D'Atri, Laboratory of Molecular Oncology, Istituto Dermopatico dell'Immacolata-IRCCS, Via dei Monti di Creta 104, I-00167 Rome, Italy

E-mail: s.datri@idi.it

Key words: microRNA, microRNA-155, melanoma, proliferation, apoptosis melanoma cells and establish for the first time that miRNA155 is a negative regulator of melanoma cell proliferation and survival.

\section{Introduction}

MicroRNAs (miRNAs) are a class of small ( 22-nt) noncoding RNAs, which play an important role in the negative regulation of gene expression (reviewed in refs. 1-5). They are present in plant and animal cells and are involved in numerous cellular processes, including apoptosis, proliferation, differentiation and metabolism (1-5).

Genes coding for miRNAs (microRNA genes, $m i R s$ ) are transcribed into primary transcripts, which are sequentially processed by the RNase III endonucleases Drosha and Dicer to release double-stranded, 22-nt long fragments (reviewed in ref. 6). One strand of the miRNA duplex is subsequently incorporated into a ribonucleoprotein complex termed miRNAinduced silencing complex. In animal cells, single-stranded miRNAs bind to specific target mRNAs through partially complementary sequences, usually in the $3^{\prime}$ untranslated region, and direct the miRNA-induced silencing complex to down-regulate gene expression by mRNA translational repression, which is frequently associated with mRNA decay (6).

There is increasing evidence that miRs can be aberrantly expressed in cancer, suggesting that they may play a role as a novel class of oncogenes or tumor suppressor genes (reviewed in refs. 7-10). However, only a limited number of investigations have addressed the role of miRNA de-regulation in melanoma onset and progression (11-22).

In the present study, we first used a sensitive real-time quantitative reverse transcription-PCR (qRT-PCR) assay to 
evaluate, in a panel of melanoma cell lines and normal melanocytes, the expression levels of seven miRNAs, namely miRNA-17-5p, miRNA-18a, miRNA-20a, miRNA-92a, miRNA-146a, miRNA-146b and miRNA-155. Actually, previous studies indicated that alterations in the expression of these miRNAs may have a role in cancer development and/or progression (7-10). Thereafter we focused our attention on miRNA-155, since it resulted markedly down-regulated in the majority of melanoma cell lines. In order to establish the possible biological significance of the low miRNA-155 expression in melanoma, we investigated the effects of the ectopic expression of the miRNA on in vitro melanoma cell proliferation and apoptosis.

\section{Materials and methods}

Cell lines and normal melanocytes. Seventeen human melanoma cell lines were used in this study and cultured as previously described (23). GR-Mel and PNP-Mel were derived from primary melanomas, whereas the other cell lines were originated from metastatic lesions.

Human melanocytes were isolated from normal skin biopsies of 10 different donors and cultured, as previously described (24).

All biological material was obtained with the patient's informed consent and the study was conducted according to the Declaration of Helsinki Principles.

Low molecular weight (LMW) RNA isolation and qRT-PCR analysis of miRNA expression. LMW RNA was isolated from melanocytes and melanoma cell lines using the mirVana ${ }^{\mathrm{TM}}$ miRNA isolation kit (Ambion, Austin, TX) according to the manufacturer's protocol. LMW RNA was quantified using the NanoDrop ND-1000 spectrophotometer (Thermo Fisher Scientific Inc, Waltham, MA).

To evaluate the expression of U6 small nuclear RNA (snRNA) and mature miRNAs, the TaqMan ${ }^{\circledR}$ MicroRNA Reverse Transcription kit, the TaqMan Universal PCR Master Mix No AmpErase ${ }^{\circledR}$ UNG and the TaqMan MicroRNA Assay for U6 snRNA and the selected miRNAs, all purchased from Applied Biosystems (Foster City, CA), were used. All experimental procedures were performed according to the manufacturer's protocols. One or $10 \mathrm{ng}$ of LMW RNA were reverse transcribed in a final volume of $15 \mu \mathrm{l}$ and qRT-PCR was done on an ABI PRISM 7000 Sequence Detection System (Applied Biosystems) in a final volume of $20 \mu \mathrm{l}$. All qRT-PCR reactions were run in duplicate. The expression of the miRNAs under investigation relative to miRNA-16 was determined

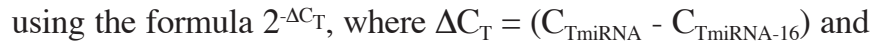
$\mathrm{C}_{\mathrm{T}}$ (i.e. threshold cycle) indicates the fractional cycle number at which the amount of amplified target reaches a fixed threshold (25).

Transfection. Pre-miR hsa-miR-155 miRNA Precursor (premiRNA-155) and Pre-miR miRNA Precursor Negative Control \#1 (dsRNA-CTRL) were obtained from Ambion.

To evaluate the effect of miRNA-155 on cell growth, melanoma cells were seeded into 24-well plates (Falcon, Becton and Dickinson Labware, Franklin Lakes, NJ) and allowed to adhere at $37^{\circ} \mathrm{C}$ for $18 \mathrm{~h}$. The cells were then transfected with pre-miRNA-155 or dsRNA-CTRL. Transfection was performed using Oligofectamine or Lipofectamine 2000 (Invitrogen Corporation, Carlsbad, CA) in serum-free medium, according to the manufacturer's protocol. Additional controls consisted in melanoma cells left untreated or exposed to the transfection reagent only (mock-transfected cells). Three replica wells were used for each group. After $72 \mathrm{~h}$ of culture, the cells were subjected to a second transfection. Seventy-two hours later, the cells were harvested by trypsinization and cell growth was evaluated in terms of viable cell count. Transfection efficiency was evaluated using a fluoresceinlabeled double-stranded RNA oligomer designated BLOCK$\mathrm{iT}^{\mathrm{TM}}$ fluorescent oligonucleotide (Invitrogen).

Evaluation of apoptosis. Melanoma cells were plated in duplicate in 24-well plates and transfected with $100 \mathrm{nM}$ premiRNA-155 or dsRNA-CTRL, as described above. Forty-eight hours after a single transfection procedure, apoptotic death was evaluated using the Cell Death Detection ELISA ${ }^{\text {PLus }}$ kit (Roche Diagnostics GmbH, Mannheim, Germany), according to the manufacturer's protocols. This kit allows the quantitative determination of mono- and oligonucleosomes that accumulate in the cytoplasm of cells undergoing apoptosis before plasma membrane breakdown. Signals were determined in a Microplate Reader 3550-UV (Bio-Rad, Hercules, CA). Data were expressed in terms of 'Enrichment Factor', calculated as the ratio between the adsorbance values of pre-miRNA-155transfected cells and those of dsRNA-CTRL-transfected cells.

Statistical analysis of qRT-PCR data. We performed two different statistical analyses to assess the significance of the differences in miRNA expression within a class of samples and between classes of samples.

The statistical differences within a class of samples were determined using a customized script employing Bioconductor packages (http://www.bioconductor.org) and based on the $\mathrm{R}$ language (http://www.r-project.org). In particular, we used 'Permtest' and 'BootPR' R-packages to perform t-test in conjunction with bootstrap analysis in order to determine which gene between U6 snRNA and miRNA-16 had the lowest variability among melanocytes. Fifty-thousand permutations were applied to the test in order to define the confidence limits and the corresponding significance thresholds. The statistical significance of the differential expression of U6 snRNA or miRNA-16 among the melanocytes was assessed by computing a P-value for each $2^{-\mathrm{CT}}$ value. No specific parametric form was assumed for the distribution of the test statistics. To determine the P-value, we used a permutation procedure in which the expression value of U6 snRNA or miRNA-16 was permuted 500,000 times, and for each permutation, two-sample tstatistics were computed for each value. The permutation $\mathrm{P}$-value for a particular value is the proportion of the permutations (out of 500,000) in which the permuted test statistic exceeds the observed test statistic in absolute values. The same statistical analysis was applied to assess variability of miRNA-16 expression within the group of melanoma cell lines.

The statistical significance of the differences in miRNA expression between melanoma cell lines and melanocytes was assessed by Student's t-test analysis performed on $2^{-\Delta \mathrm{CT}}$ values. 


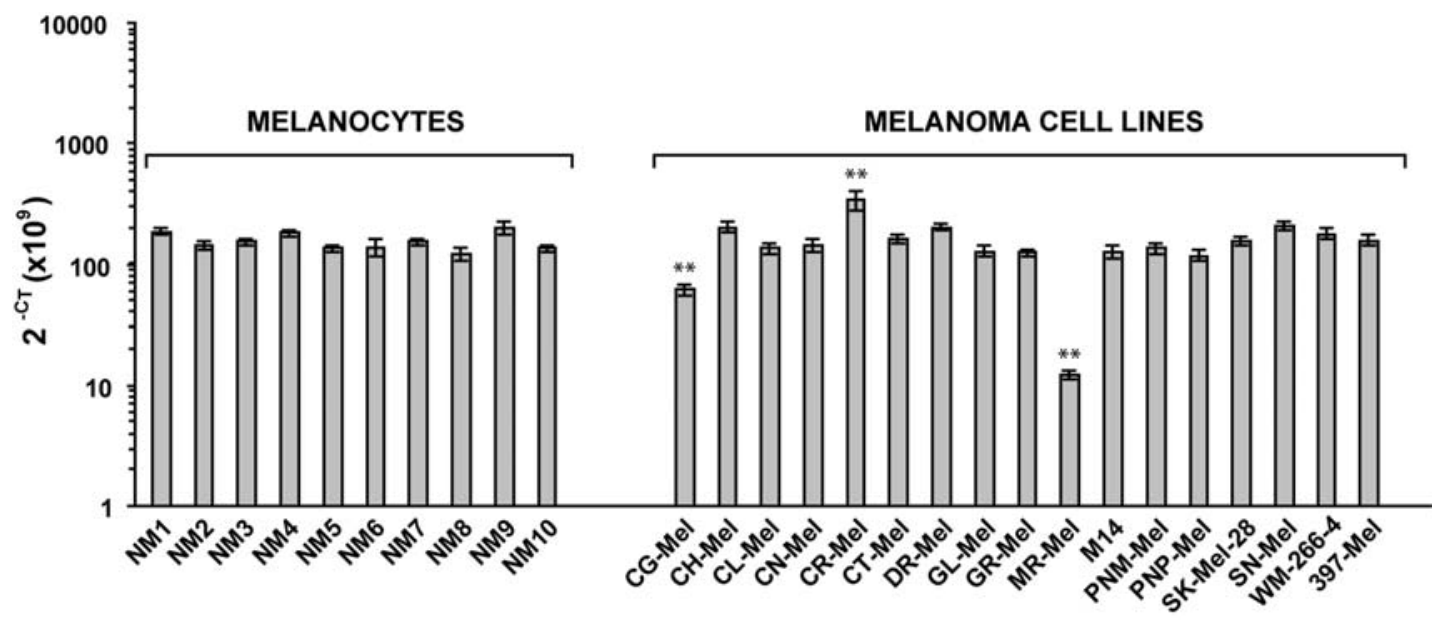

Figure 1. Expression levels of miRNA-16 in normal melanocytes and melanoma cell lines. Equal amounts (1.33 $\mu 1)$ of cDNA reverse transcribed from 1 ng of LMW RNA were used to determined by qRT-PCR the expression of miRNA-16 in normal skin melanocytes obtained from 10 different donors and in 17 melanoma cell lines. Data are expressed in terms of $2^{-\mathrm{CT}} \times 10^{9}$. Each value represents the mean of six independent experiments, in which qRT-PCRs were run in duplicate. Bars, standard error of the mean. Among the melanoma group, three cell lines were found to be outliers $\left({ }^{* *} \mathrm{P}<0.01\right.$, according to t-test in conjunction with bootstrap analysis). The mean expression value for the melanocyte group was $155 \pm 8.13$ and the mean expression value for the melanoma cell line group, not including CG-Mel, CR-Mel and MR-Mel, was 155 \pm 8.25 .

\section{Results}

Selection of the internal control gene for the evaluation of miRNA expression by $q R T-P C R$. Previous studies showed that melanin inhibits RT-PCR (26). Therefore, to reduce melanin contamination and to enrich the miRNA content, we used column purified LMW RNA for qRT-PCR. U6 snRNA and miRNA-16 have been previously used as internal control genes to determine the relative expression of a large number of miRNAs in cell lines by qRT-PCR performed on total RNA $(11,27)$. Therefore, preliminary experiments were carried out to select, between the two genes, the best internal control to be adopted for the present study.

The first set of experiments showed that the $2^{\text {-CT }}$ value of miRNA-16 was significantly higher $(\mathrm{P}<0.01$ according to Student's t-test) than that of U6 snRNA in melanocytes isolated from normal skin biopsies of 10 different donors (data not shown). Moreover, t-test in conjunction with bootstrap analysis showed that variability of U6 snRNA expression among the different melanocyte samples was higher than that of miRNA-16 (i.e. $\mathrm{P}<0.01$ or $\mathrm{P}<0.05$ in 8 out of 10 samples in the case of U6 snRNA vs. no statistically significant variation in the case of miRNA-16, data not shown).

In a subsequent set of experiments, the expression level of miRNA-16, in terms of $2^{-\mathrm{CT}}$ value, was determined in parallel in the 10 melanocyte samples and in 17 melanoma cell lines. The results of t-test in conjunction with bootstrap analysis confirmed that no significant differences existed in the expression of miRNA-16 within the group of melanocytes (Fig. 1). The same statistical analysis applied to melanoma cell lines identified three outliers (Fig. 1), indicating that deregulation of miRNA-16 can occur in some melanomas. These 3 cell lines were then excluded from further analysis. Student's t-test analysis performed on miRNA-16 2-CT values relative to the remaining 14 melanoma cell lines and to the melanocytes showed no significant difference (Fig. 1). On these bases we selected miRNA-16 as the internal control gene for qRT-PCR assays.
Expression of mature miRNAs in normal cultured melanocytes and melanoma cell lines. All qRT-PCR assays were performed using $1.33 \mu \mathrm{l}$ of cDNA reverse transcribed from $1 \mathrm{ng}$ of LMW RNA. However, the levels of miRNA-155 were found to be extremely low in melanoma cells. Therefore, the expression of this miRNA was evaluated using $1.33 \mu \mathrm{l}$ of cDNA reverse transcribed from 10 ng of LMW RNA.

As illustrated in Table I, all melanocyte samples expressed the miRNAs under investigation, being miRNA-146a the most expressed and miRNA-18a the less expressed miRNA in all samples.

To identify miRNAs dysregulated in melanoma cell lines with respect to melanocytes, we performed Student's t-test analysis between the two groups (i.e. all melanoma cell lines vs. all melanocytes) and between each melanoma cell line and the group of melanocytes. When melanoma cell lines and melanocytes were compared as two groups, miRNA-17-5p, miRNA-18a, and miRNA-20a, which are encoded by the miR-17-92 cluster, resulted overexpressed in the melanoma group (Table I). miRNA-92a, which is generated from the transcription of two different miRs (i.e. miR-92a-1 in the $m i R-17-92$ cluster and $m i R-92 a-2$, in the $m i R-106 a-363$ cluster) (reviewed in ref. 28) was also up-regulated in the melanoma group (Table I). In contrast, the expression of miRNA-146a, miRNA-146b and miRNA-155, was significantly reduced in the melanoma group with respect to that of melanocytes (Table I).

When each melanoma cell line was compared with the melanocyte group, the four miRNAs encoded by the miR-17-92 cluster were up-regulated simultaneously in 9 cell lines. In contrast, miRNA-146a and miRNA-146b, were found to be concomitantly down-regulated in 10 melanomas and miRNA-155 resulted to be significantly reduced in 13 cell lines (Table I).

Transfection of pre-miRNA-155 into melanoma cell lines inhibits proliferation. To investigate the biological significance of miRNA-155 down-regulation in melanoma cells, we decided 
Table I. Expression of miRNAs in human normal melanocytes and melanoma cell lines.

\begin{tabular}{|c|c|c|c|c|c|c|c|}
\hline \multirow[t]{2}{*}{ miRNA } & \multicolumn{3}{|c|}{ Relative expression level $\left(2^{-\Delta \mathrm{CT}}\right)^{\mathrm{a}}$} & \multicolumn{2}{|c|}{$\begin{array}{l}\text { Melanoma cell lines showing } \\
\text { up-regulation of the miRNA }\end{array}$} & \multicolumn{2}{|c|}{$\begin{array}{l}\text { Melanoma cell lines showing } \\
\text { down-regulation of the miRNA }\end{array}$} \\
\hline & Melanocytes & Melanoma & $\mathrm{P}^{\mathrm{b}}$ & No. ${ }^{c}$ & MC:NM Ratio & No. ${ }^{c}$ & MC:NM Ratiod \\
\hline $17-5 p$ & $0.045 \pm 0.002$ & $0.132 \pm 0.020$ & $<0.01$ & 10 & $2.3-5.5$ & 0 & - \\
\hline $18 \mathrm{a}$ & $0.016 \pm 0.001$ & $0.049 \pm 0.008$ & $<0.01$ & 10 & $2.1-6.4$ & 0 & - \\
\hline $20 \mathrm{a}$ & $0.247 \pm 0.010$ & $0.449 \pm 0.054$ & $<0.01$ & 11 & $1.5-3.4$ & 2 & $0.7-0.6$ \\
\hline $92 \mathrm{a}$ & $0.227 \pm 0.018$ & $0.367 \pm 0.041$ & $<0.01$ & 10 & $1.3-3.1$ & 1 & 0.5 \\
\hline $146 a$ & $2.057 \pm 0.142$ & $1.189 \pm 0.169$ & $<0.01$ & 0 & - & 10 & $0.8-0.2$ \\
\hline $146 b$ & $1.770 \pm 0.144$ & $0.861 \pm 0.140$ & $<0.01$ & 0 & - & 10 & $0.6-0.1$ \\
\hline 155 & $0.069 \pm 0.011$ & $0.005 \pm 0.004$ & $<0.01$ & 0 & - & 13 & $0.2-0.0004$ \\
\hline
\end{tabular}

${ }^{a}$ The expression of miRNAs was determined by qRT-PCRs in melanocytes obtained from 10 different donors and in 14 melanoma cell lines. Values represent the mean \pm standard error of the mean of the melanocyte or the melanoma group. For each melanocyte sample and melanoma cell line at least three independent experiments, in which qRT-PCRs were run in duplicate, were performed. bP, probability according to Student's t-analysis comparing miRNA expression values of melanocytes with those of melanoma cell lines. ${ }^{\mathrm{N}}$ Number of melanoma cell lines in which miRNA expression value was significantly higher or lower ( $\mathrm{P}<0.05$ according to Student's t-test analysis) than the mean expression value of the melanocyte group. ${ }^{\mathrm{d}}$ Range of the ratio between the mean expression value of each melanoma cell line (MC) and the mean expression value of the melanocyte group (NM).

to assess the effect of ectopic expression of the miRNA on cell proliferation. To this end, all the melanoma cell lines were left untreated, mock-transfected or subjected to two sequential transfection procedures with $100 \mathrm{nM}$ of a double-stranded RNA mimicking the endogenous precursor of miRNA-155 (pre-miRNA-155) or with a double-stranded control RNA (dsRNA-CTRL). Cell growth was evaluated in terms of viable cell count $72 \mathrm{~h}$ after the second transfection.

In preliminary experiments, the cell lines were transfected with either Oligofectamine or Lipofectamine 2000 and the BLOCK-iT ${ }^{\mathrm{TM}}$ fluorescent oligonucleotide $(100 \mathrm{nM})$ and assayed for transfection efficiency after $24 \mathrm{~h}$. The cell lines were also subjected to two sequential transfections with the transfection reagents alone and assayed for proliferation $72 \mathrm{~h}$ after the second transfection. Based on the results of this set of experiments (data not shown), in 13 out of 14 cell lines we were able to select for the transfection reagent providing an acceptable transfection efficiency and minimal effects on proliferation (Table II and Fig. 2). In the remaining cell line (i.e. CT-Mel), we used Lipofectamine 2000 despite its marked inhibitory effect on cell growth because transfection efficiency with Oligofectamine was only $10 \%$.

The effects of pre-miRNA-155 transfection on melanoma cell growth are illustrated in Fig. 2. In 12 melanoma cell lines, growth inhibition induced by transfection of pre-miRNA-155 was significantly higher than that observed in dsRNA-CTRLtransfected cells, with percentages of cell growth inhibition ranging between 30 and $98 \%$. In the remaining cell lines (i.e. GR-Mel and PNM-Mel), no increase of cell growth inhibition was induced by pre-miRNA-155 transfection with respect to dsRNA-CTRL. Notably, GR-Mel, was the only cell line showing miRNA-155 levels comparable to those of melanocytes.

To further assess the inhibitory activity of miRNA-155 on melanoma cell growth, a concentration-response curve was set up with CH-Mel, DR-Mel, GL-Mel and SK-Mel-28 cell lines.

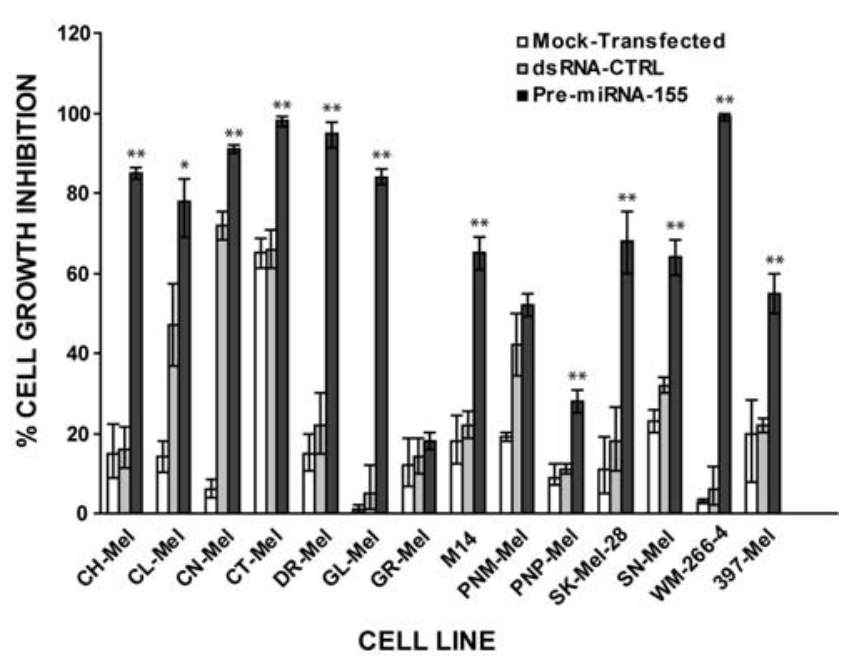

Figure 2. Ectopic expression of miRNA-155 inhibits melanoma cell growth. Melanoma cells were left untreated, mock-transfected or subjected to two sequential transfections with $100 \mathrm{nM}$ pre-miRNA-155 or dsRNA-CTRL, as described under Materials and methods. Seventy-two hours after the second transfection, the cells were harvested by trypsinization and cell growth was evaluated in terms of viable cell count. Data are expressed in terms of percentage of growth inhibition of target cells transfected with pre-miRNA155, or dsRNA-CTRL or mock-transfected with respect to the untreated cells. Each value represents the mean of at least three independent experiments performed with triplicate samples, with bars indicating standard error of the mean. ${ }^{* *} \mathrm{P}<0.01$, according to Student's t-test, comparing the percentages of cell growth inhibition of pre-miRNA-155-transfected cells with those of dsRNA-CTRL-transfected cells. Percentages were subjected to angular transformation in order to obtain normally distributed data. Thereafter, conventional standard error calculation and Student's t-test statistics were performed on converted data. However, the data are expressed in non-transformed percentages, following conversion of transformed data into the original values.

The results illustrated in Fig. 3 show that pre-miRNA-155, but not dsRNA-CTRL induced a concentration-dependent inhibition of cell growth. 
Table II. Transfection efficiency and cell growth inhibition relative to the transfection reagent selected for melanoma cell lines.

\begin{tabular}{|c|c|c|c|}
\hline Transfection reagent & Cell line & $\begin{array}{c}\text { Transfection efficiency } \\
\text { Range }(\%)\end{array}$ & $\begin{array}{c}\text { Cell growth inhibition }{ }^{\mathrm{b}} \\
\text { Range }(\%)\end{array}$ \\
\hline Oligofectamine & $\begin{array}{c}\text { CH-Mel } \\
\text { CN-Mel } \\
\text { DR-Mel } \\
\text { GL-Mel } \\
\text { WM-266-4 }\end{array}$ & $\begin{array}{l}80-90 \\
80-90 \\
80-90 \\
60-70 \\
70-80\end{array}$ & $\begin{array}{c}10-20 \\
5-10 \\
10-20 \\
<5 \\
<5\end{array}$ \\
\hline Lipofectamine 2000 & $\begin{array}{c}\text { CL-Mel } \\
\text { CT-Mel } \\
\text { GR-Mel } \\
\text { M14 } \\
\text { PNM-Mel } \\
\text { PNP-Mel } \\
\text { SK-Mel-28 } \\
\text { SN-Mel } \\
\text { 397-Mel }\end{array}$ & $\begin{array}{c}80-90 \\
90-100 \\
70-80 \\
70-80 \\
90-100 \\
80-90 \\
70-80 \\
80-90 \\
80-90\end{array}$ & $\begin{array}{c}10-20 \\
60-70 \\
5-20 \\
15-25 \\
15-20 \\
5-15 \\
5-20 \\
15-25 \\
15-25\end{array}$ \\
\hline
\end{tabular}

${ }^{\mathrm{a}}$ Melanoma cells were seeded into 24-well plates, allowed to adhere at $37^{\circ} \mathrm{C}$ for $18 \mathrm{~h}$ and then transfected with $100 \mathrm{nM} \mathrm{BLOCK}-\mathrm{iT} \mathrm{T}^{\mathrm{TM}}$ fluorescent oligonucleotide using Oligofectamine or Lipofectamine 2000. Transfection efficiency was evaluated $24 \mathrm{~h}$ after transfection using a fluorescence microscope (Axiovert 135, Zeiss, Oberkochen, Germany). belanoma cells were subjected to two sequential transfection procedures with the transfection reagent alone, as described in Materials and methods. Cell proliferation was evaluated $72 \mathrm{~h}$ after the second transfection.

Transfection of pre-miRNA-155 into melanoma cell lines induces apoptosis. To investigate whether the growth inhibitory effect of pre-miRNA-155 could be due, at least in part, to the triggering of apoptosis, experiments were performed on 4 different cell lines, that were subjected to a single transfection with $100 \mathrm{nM}$ pre-miRNA-155 or dsRNA-CTRL and assayed for apoptosis $48 \mathrm{~h}$ later. Ectopic expression of miRNA-155 was able to induce apoptosis in all the 4 cell lines tested (Fig. 4). Moreover, apoptosis was particularly pronounced in the two cell lines in which transfection with pre-miRNA-155 was followed by strong cell growth suppression (i.e. $\mathrm{CH}-\mathrm{Mel}$ and DR-Mel).

\section{Discussion}

Aberrant expression of the miR-17-92 cluster or single components of the cluster, as well as of $m i R-146 a, m i R-146 b$ and $m i R-155$ can have a role in tumorigenesis $(7,8,10,28)$. To investigate whether these miRs could be deregulated in melanoma, we comparatively analyzed the expression levels of the corresponding mature miRNAs in a panel of human melanoma cell lines and cultured normal melanocytes. Notably, the qRT-PCR assays were performed on RNA preparations enriched for miRNAs, with conceivably reduced content of melanin. Moreover, the internal control gene (i.e. miRNA-16) was selected following an experimental survey of two candidate small RNA molecules largely used as reference gene transcripts. We found that miRNA-17-5p, miRNA-18a, miRNA-20a, and miRNA-92a were up-regulated, whereas miRNA-146a, miRNA-146b and miRNA-155 were downregulated in the majority of the melanoma cell lines analyzed.
We focused our attention on the biological role of miRNA155 , that was found, for the first time, to be a candidate gene able to control melanoma cell growth and survival. Indeed, we observed that enforced expression of miRNA-155 was able to inhibit proliferation and induce apoptosis in melanoma cells expressing low levels of this miRNA.

In humans, $m i R-155$ resides within the BIC gene on chromosome $21(7,8)$. High levels of miRNA-155 have been found in B-CLL, B-cell lymphomas, papillary tyroid carcinoma, breast cancer (7-10), pancreatic ductal adenocarcinoma (29) and other tumors (30). Moreover, enforced expression of miRNA-155 is sufficient to trigger murine B lymphoma (7). On the other hand, miRNA-155 was reported to be expressed in healthy pancreas and essentially absent in endocrine pancreatic tumors (10). Moreover, the levels of this miRNA were found to be reduced in ovarian cancer (31). These findings suggest that $m i R-155$ can act either as oncogene or as tumor suppressor gene, depending on the cell background in which miRNA-155 is performing its specific target gene controlling function.

Our results demonstrate that miRNA-155, which appears to be the most altered miRNA among those analyzed, is a negative regulator of melanoma cell growth. Actually, ectopic expression of miRNA-155 significantly inhibited proliferation in 12 out of 13 melanoma cell lines endowed with low miRNA-155 levels. In contrast, enforced expression of this miRNA did not affect the growth of GR-Mel cells, which display miRNA-155 levels comparable to those of melanocytes. It must be noted that the transfection efficiency of GR-Mel cells was $\sim 70-80 \%$, thus eliminating the possibility that ineffective pre-miRNA-155 uptake might underlie the lack of response. Moreover, a concentration-dependent inhibition 
a

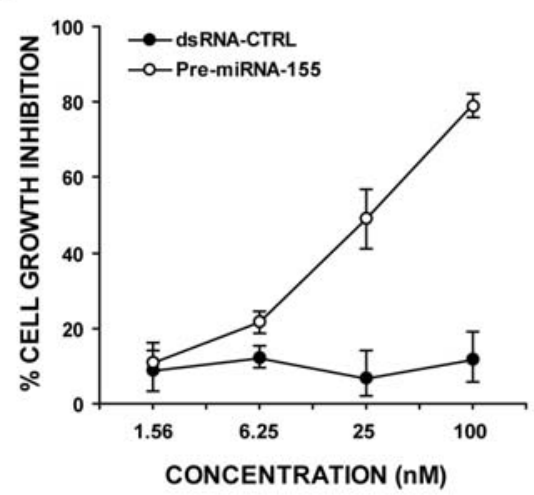

C

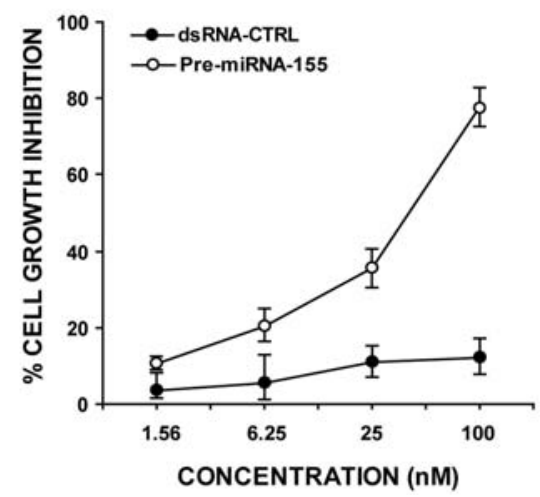

b

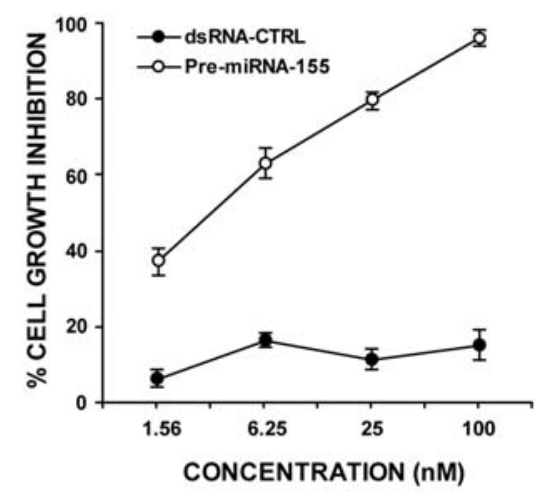

d

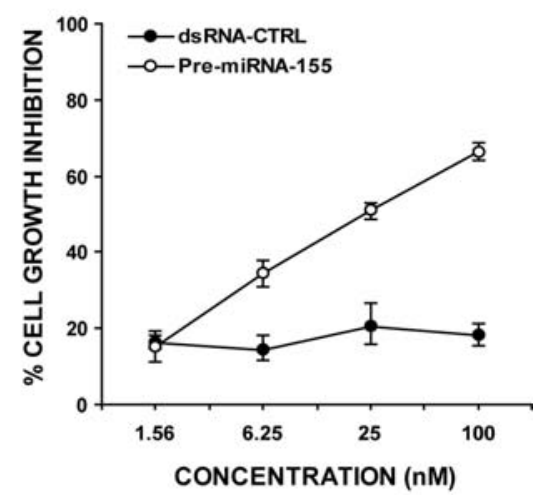

Figure 3. Ectopic expression of miRNA-155 induces a concentration-dependent inhibition of melanoma cell growth. CH-Mel (a), DR-Mel (b), GL-Mel (c) and SK-Mel-28 (d) cells were left untreated or subjected to two sequential transfections with the indicated concentrations of pre-miRNA-155 or dsRNA-CTRL, as describe under Materials and methods. Seventy-two hours after the second transfection, the cells were harvested by trypsinization and cell growth was evaluated in terms of viable cell count. Data are expressed in terms of percentage of growth inhibition of target cells transfected with pre-miRNA-155 or dsRNA-CTRL with respect to untreated cells. Each value represents the mean of at least three independent experiments performed with triplicate samples, with bars indicating standard error of the mean. Percentages were subjected to angular transformation in order to obtain normally distributed data. Thereafter, conventional standard error calculation was performed on converted data. However, the data are expressed in non-transformed percentages, following conversion of transformed data into the original values.

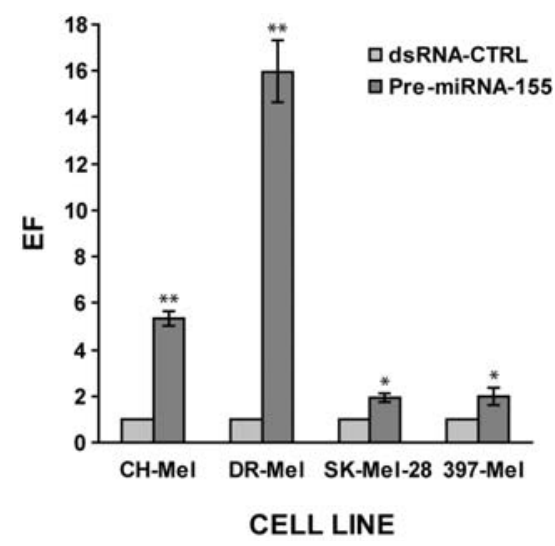

Figure 4. Ectopic expression of miRNA-155 induces apoptosis in melanoma cells. CH-Mel, DR-Mel, SK-Mel-28 and 397-Mel cells were subjected to a single transfection procedure with $100 \mathrm{nM}$ pre-miRNA-155 or dsRNA-CTRL, as described in Materials and methods. Forty-eight hours after transfection, the cytoplasmic amount of mono- and oligonucleosomes originated from apoptotic DNA degradation was quantified using an ELISA assay. Data are expressed in terms of Enrichment Factor (EF), calculated as the ratio between the adsorbance value of pre-miRNA-155-transfected cells and that of dsRNA-CTRL-transfected cells, to which the arbitrary value of 1.0 was assigned. Each value represents the mean of at least four independent experiments. Bars, standard error of the mean. ${ }^{* *} \mathrm{P}<0.01$ and ${ }^{*} \mathrm{P}<0.05$, according to Student's t-test, comparing the adsorbance values of premiRNA-155-transfected cells with those of dsRNA-CTRL-transfected cells. of cell growth was observed upon transfection of pre-miRNA155 but not of dsRNA-CTRL, thus further supporting the specificity of the inhibitory effects of miRNA-155. Impairment of melanoma cell proliferation appears to be dependent, at least in part, on miRNA-155-mediated induction of apoptosis. Indeed, in 4 out of 4 cell lines tested ectopic expression of miRNA-155 caused significantly higher levels of apoptosis than ectopic expression of dsRNA-CTRL.

Since miRNAs can regulate a large number of target genes, several algorithms have been developed to predict in silico the targets of selected miRNAs. We adopted the TargetScan (http://www.targetscan.org/) and PicTar (http://www.pictar. org/) algorithms to identify putative miRNA-155 targets and found that 160 targets are predicted by both algorithms. Down-regulation of one or more of these target genes might be involved in miRNA-155-induced impairment of melanoma cell proliferation and survival. For instance, the MAP3K14 genes code for nuclear factor-inducing kinase (NIK), which plays a central role in the activation of the non-canonical $\mathrm{NF}-\kappa \mathrm{B}$ pathway in response to a subset of NF- $\mathrm{BB}$-inducing stimuli (reviewed in ref. 32). It has been shown that NIK expression and/or activity is significantly higher in melanoma cells than in normal melanocytes and that overexpression of a kinase-deficient mutant of NIK strongly reduces basal NF- $\mathrm{KB}$ 
activity in melanoma cells but not in melanocytes (33). Inhibition of $N F-\kappa B$ activity in melanoma cells through several strategies has been shown to exert antitumor effects both in vitro and in vivo (33). On these bases, it is possible to speculate that inhibition of NIK expression could be involved in the antiproliferative effects of miRNA-155 in melanoma.

Another potential target gene that might be involved in the growth inhibitory effects of miRNA-155 in melanoma cells is SKI. This gene encodes a transcriptional co-regulator that interacts with several proteins, including the retinoblastoma protein, SMAD2, SMAD3, SMAD4, the SKI-interacting protein SKIP, FHL2, mSin3 and N-coR (reviewed in ref. 34). $S K I$ transcripts are much more expressed in melanoma cell lines than in normal melanocytes (34) and high expression of the SKI protein has been reported in melanoma specimens (34). Moreover, it was demonstrated that down-regulation of SKI protein levels by antisense $S K I$ vectors or small interfering RNAs against $S K I$ inhibits melanoma cell proliferation and clonogenicity (34), while increased SKI dosage stimulates melanoma cell proliferation (35).

In any case, a large amount of experimental study is still ahead to definitively establish the main gene transcripts actually targeted by miRNA-155 and involved in the unexpectedly onco-suppressive effects of this $m i R$ gene in melanoma.

The polycistronic $m i R-17-92$ cluster resides in the C13orf 25 gene locus on chromosome 13 and comprises six miRs, i.e. $m i R-17$ (which codes for miRNA-17-5p and miRNA-17-3p), miR18a, miR-19a, miR20a, miR-19b-1 and miR-92a-1 (28). The expression of the entire cluster, or of single components of it, has been shown to be frequently altered in different types of cancer and experimental evidence indicates that members of the cluster can have both oncogenic and tumor suppressive functions (28). For instance, the $m i R-17-92$ polycistron is up-regulated in $65 \%$ of B-cell lymphoma samples and enforced expression of the cluster accelerates $M Y C$-induced lymphomagenesis (28). On the other hand, reduced expression of miRNA-17-5p and miRNA-20a has been reported in breast cancer cell lines and specimens $(30,36,37)$. Consistently, ectopic expression of miRNA-17-5p and miRNA-20a was found to negatively regulate breast cancer cell proliferation (36,37).

A previous study performed by Zhang et al (11) on 45 primary cultured melanoma cell lines reported a copy number loss of the region containing the miR-17-92 cluster in $\sim 20 \%$ of the melanoma cell lines analyzed, suggesting that the cluster may act as a tumor suppressor gene in melanoma. However, a very recent investigation (22), in which miRNA expression profiles were detemined in cultured melanocytes and several melanoma cell lines using Agilent miRNA microarrays, showed up-regulation of the miR-17-92 cluster in the melanoma cell lines. This finding suggests an oncogenic role of the cluster in melanoma.

Our data, obtained using qRT-PCR assays, which are considered the gold standard for RNA quantification, confirm the findings of Wilhelm Mueller et al (22) in a different panel of melanoma cell lines and in a higher number of melanocytic cultures. Indeed, the four components of the miR-17-92 cluster that have been tested, resulted simultaneously overexpressed in 9 out of 14 melanomas. It is possible to hypothesize that in these melanomas, the entire $m i R-17-92$ cluster is overexpressed as a results of locus amplification or increased transcription of the polycistron. Interestingly, the expression of the miR-17-92 cluster is activated by the transcription factor MYC (28) and $M Y C$ has been shown frequently to be overexpressed or amplified in melanoma (38).

Presently, several genes have been experimentally identified as targets of members of the miR-17-92 cluster. These genes include $E 2 F 1, E 2 F 2, E 2 F 3$, coding for transcription factors involved in cell cycle regulation, $B C L 2 L 11$, coding for the pro-apoptotic molecule Bim, $C D K N 1 A$, coding for the cyclin-dependent kinase inhibitor p21, as well as THBS1 and $C T G F$, coding for the antiangiogenic proteins thrombospondin- 1 and connective tissue growth factor, respectively (28). Down-regulation of these target genes might contribute to melanoma development and/or progression, thus supporting the hypothesis that $m i R-17-92$ cluster is endowed with oncogenic activity.

The human genome contains two miR-146 genes, i.e. $m i R-146 a$ on chromosome 5 and $m i R-146 b$ on chromosome 10 (39). Previous studies suggest a possible role of $m i R-146 a$ and $m i R-146 b$ as oncogenes or tumor suppressor genes, depending on the cell lineage $(8,10,11,30,40,41)$. In particular, a copy number loss of the region containing miR-146b was detected by Zhang et al (11) in 24\% of melanoma cell lines.

Our data show a concomitant and statistically significant down-regulation of miRNA-146a and miRNA-146b levels in 10 out of 14 melanoma cell lines. Moreover, in most melanoma cell lines the levels of the two miRNAs were found to be $<50 \%$, a threshold usually considered to be of biological relevance. Based also on the study by Zhang et al (11) it is therefore possible to speculate that in cells of the melanocytic lineage down-regulation of miRNA-146a and miRNA-146b could promote malignant transformation and/or tumor progression. Noteworthy, the finding that miRNA-146a and miRNA-146b are similarly deregulated in melanoma cells suggests a coordinate functional role and possibly responsiveness to a common upstream regulator. Interestingly, it was recently shown that the expression of $m i R-146 a$ is directly repressed by MYC (42), opening up the possibility that down-regulation of miRNA-146a and miRNA-146b in melanoma cells could be, at least in part, associated with $M Y C$ overexpression.

In conclusion, our study demonstrates that overexpression of miRNAs encoded by the miR-17-92 cluster and downregulation of miRNA-146a, miRNA-146b and miRNA-155 are frequent events in melanoma cell lines, supporting the involvement of these miRNAs in melanocyte malignant transformation and/or in melanoma progression. Moreover, our results show, for the first time, that miRNA-155 can be classified as a negative regulator of melanoma cell proliferation and survival. The experimental identification of miRNA-155 target genes might help, in the long-term, to set up novel treatment strategies to cope with the still unresolved problem of an effective control of advanced melanoma.

\section{Acknowledgements}

This study was supported in part by a grant from Istituto Superiore di Sanità, Italy-USA Oncology Program, contract 
527B/6 and in part by a grant from the Italian Ministry of Health, contract RF2005/68.

\section{References}

1. Bushati N and Cohen SM: MicroRNA functions. Annu Rev Cell Dev Biol 23: 175-205, 2007.

2. Niwa R and Slack FJ: The evolution of animal microRNA function. Curr Opin Genet Dev 17: 145-150, 2007.

3. Zhang B, Wang Q and Pan X: MicroRNAs and their regulatory roles in animals and plants. J Cell Physiol 210: 279-289, 2007.

4. Zhao Y and Srivastava D: A developmental view of microRNA function. Trends Biochem Sci 32: 189-197, 2007.

5. Williams AE: Functional aspects of animal microRNAs. Cell Mol Life Sci 65: 545-562, 2008.

6. Filipowicz W, Bhattacharyya SN and Sonenberg N: Mechanisms of post-transcriptional regulation by microRNAs: are the answers in sight? Nat Rev Genet 9: 102-114, 2008.

7. Calin GA and Croce CM: MicroRNA signatures in human cancers. Nat Rev Cancer 6: 857-866, 2006.

8. Esquela-Kerscher A and Slack FJ: Oncomirs - microRNAs with a role in cancer. Nat Rev Cancer 6: 259-269, 2006.

9. Barbarotto E, Schmittgen TD and Calin GA: MicroRNAs and cancer: profile, profile, profile. Int J Cancer 122: 969-977, 2008.

10. Gartel AL and Kandel ES: miRNAs: Little known mediators of oncogenesis. Semin Cancer Biol 18: 103-110, 2008.

11. Zhang L, Huang J, Yang N, Greshock J, Megraw MS, Giannakakis A, Liang S, Naylor TL, Barchetti A, Ward MR, Yao G, Medina A, O'Brien-Jenkins A, Katsaros D, Hatzigeorgiou A, Gimotty PA, Weber BL and Coukos G: MicroRNAs exhibit high frequency genomic alterations in human cancer. Proc Natl Acad Sci USA 103: 9136-9141, 2006.

12. Bemis LT, Chen R, Amato CM, Classen EH, Robinson SE, Coffey DG, Erickson PF, Shellman YG and Robinson WA: MicroRNA-137 targets microphthalmia-associated transcription factor in melanoma cell lines. Cancer Res 68: 1362-1368, 2008.

13. Felicetti F, Errico MC, Bottero L, Segnalini P, Stoppacciaro A, Biffoni M, Felli N, Mattia G, Petrini M, Colombo MP, Peschle C and Carè A: The promyelocytic leukemia zinc finger-microRNA$221 /-222$ pathway controls melanoma progression through multiple oncogenic mechanisms. Cancer Res 68: 2745-2754, 2008.

14. Lodygin D, Tarasov V, Epanchintsev A, Berking C, Knyazeva T, Korner H, Knyazev P, Diebold J and Hermeking H: Inactivation of miR-34a by aberrant $\mathrm{CpG}$ methylation in multiple types of cancer. Cell Cycle 7: 2591-2600, 2008.

15. Migliore C, Petrelli A, Ghiso E, Corso S, Capparuccia L, Eramo A, Comoglio PM and Giordano S: MicroRNAs impair MET-mediated invasive growth. Cancer Res 68: 10128-10136, 2008.

16. Molnar V, Tamasi V, Bakos B, Wiener Z and Falus A: Changes in miRNA expression in solid tumors: an miRNA profiling in melanomas. Semin Cancer Biol 18: 111-122, 2008.

17. Muller DW and Bosserhoff AK: Integrin beta 3 expression is regulated by let-7a miRNA in malignant melanoma. Oncogene 27: 6698-6706, 2008.

18. Schultz J, Lorenz P, Gross G, Ibrahim S and Kunz M: MicroRNA let-7b targets important cell cycle molecules in malignant melanoma cells and interferes with anchorage-independent growth. Cell Res 18: 549-557, 2008.

19. Worley LA, Long MD, Onken MD and Harbour JW: MicroRNAs associated with metastasis in uveal melanoma identified by multiplexed microarray profiling. Melanoma Res 18: 184-190, 2008.

20. Yan D, Zhou X, Chen X, Hu D, Dong XE, Wang J, Lu F, Tu L and Qu J: MicroRNA-34a inhibits uveal melanoma cell proliferation and migration through downregulation of c-Met. Invest Ophthalmol Vis Sci (In press).

21. Igoucheva O and Alexeev V: MicroRNA-dependent regulation of cKit in cutaneous melanoma. Biochem Biophys Res Commun 379: 790-794, 2009.

22. Wilhelm Mueller DW, Rehli M and Bosserhoff AK: miRNA expression profiling in melanocytes and melanoma cell lines reveals miRNAs associated with formation and progression of malignant melanoma. J Invest Dermatol (In press).
23. Lacal PM, Failla CM, Pagani E, Odorisio T, Schietroma C, Falcinelli S, Zambruno G and D'Atri S: Human melanoma cells secrete and respond to placenta growth factor and vascular endothelial growth factor. J Invest Dermatol 115: 1000-1007, 2000.

24. Bondanza S, Maurelli R, Paterna P, Migliore E, Giacomo FD, Primavera G, Paionni E, Dellambra E and Guerra L: Keratinocyte cultures from involved skin in vitiligo patients show an impaired in vitro behaviour. Pigment Cell Res 20: 288-300, 2007.

25. Livak KJ and Schmittgen TD: Analysis of relative gene expression data using real-time quantitative PCR and the $2-\Delta \Delta C_{T}$ method. Methods 25: 402-408, 2001.

26. Satyamoorthy K, Li G, Van Belle PA, Elder DE and Herlyn M: A versatile method for the removal of melanin from ribonucleic acids in melanocytic cells. Melanoma Res 12: 449-452, 2002.

27. Jiang J, Lee EJ, Gusev Y and Schmittgen TD: Real-time expression profiling of microRNA precursors in human cancer cell lines. Nucleic Acids Res 33: 5394-5403, 2005.

28. Mendell JT: miRiad roles for the miR-17-92 cluster in development and disease. Cell 133: 217-222, 2008.

29. Lee EJ, Gusev Y, Jiang J, Nuovo GJ, Lerner MR, Frankel WL, Morgan DL, Postier RG, Brackett DJ and Schmittgen TD: Expression profiling identifies microRNA signature in pancreatic cancer. Int J Cancer 120: 1046-1054, 2007.

30. Volinia S, Calin GA, Liu CG, Ambs S, Cimmino A, Petrocca F, Visone R, Iorio M, Roldo C, Ferracin M, Prueitt RL, Yanaihara N, Lanza G, Scarpa A, Vecchione A, Negrini M, Harris CC and Croce CM: A microRNA expression signature of human solid tumors defines cancer gene targets. Proc Natl Acad Sci USA 103: 2257-2261, 2006.

31. Dahiya N, Sherman-Baust CA, Wang TL, Davidson B, Shih Ie M, Zhang Y, Wood W III, Becker KG and Morin PJ: MicroRNA expression and identification of putative miRNA targets in ovarian cancer. PLoS ONE 3: e2436, 2008.

32. Dejardin E: The alternative NF-kappaB pathway from biochemistry to biology: pitfalls and promises for future drug development. Biochem Pharmacol 72: 1161-1179, 2006.

33. Amiri KI and Richmond A: Role of nuclear factor-kappa B in melanoma. Cancer Metastasis Rev 24: 301-313, 2005.

34. Reed JA, Lin Q, Chen D, Mian IS and Medrano EE: SKI pathways inducing progression of human melanoma. Cancer Metastasis Rev 24: 265-272, 2005.

35. Chen D, Xu W, Bales E, Colmenares C, Conacci-Sorrell M, Ishii S, Stavnezer E, Campisi J, Fisher DE, Ben-Ze'ev A and Medrano EE: SKI activates Wnt/beta-catenin signaling in human melanoma. Cancer Res 63: 6626-6634, 2003.

36. Hossain A, Kuo MT and Saunders GF: Mir-17-5p regulates breast cancer cell proliferation by inhibiting translation of AIB1 mRNA. Mol Cell Biol 26: 8191-8201, 2006.

37. Yu Z, Wang C, Wang M, Li Z, Casimiro MC, Liu M, Wu K, Whittle J, Ju X, Hyslop T, McCue P and Pestell RG: A cyclin D1/microRNA $17 / 20$ regulatory feedback loop in control of breast cancer cell proliferation. J Cell Biol 182: 509-517, 2008.

38. Rodolfo M, Daniotti M and Vallacchi V: Genetic progression of metastatic melanoma. Cancer Lett 214: 133-147, 2004.

39. Bentwich I, Avniel A, Karov Y, Aharonov R, Gilad S, Barad O, Barzilai A, Einat P, Einav U, Meiri E, Sharon E, Spector Y and Bentwich Z: Identification of hundreds of conserved and nonconserved human microRNAs. Nat Genet 37: 766-770, 2005.

40. Calin GA, Liu CG, Sevignani C, Ferracin M, Felli N, Dumitru CD, Shimizu M, Cimmino A, Zupo S, Dono M, Dell'Aquila ML, Alder H, Rassenti L, Kipps TJ, Bullrich F, Negrini M and Croce CM: MicroRNA profiling reveals distinct signatures in B cell chronic lymphocytic leukemias. Proc Natl Acad Sci USA 101: 11755-11760, 2004.

41. Lin SL, Chiang A, Chang D and Ying SY: Loss of mir-146a function in hormone-refractory prostate cancer. RNA 14: 417-424, 2008.

42. Chang TC, Yu D, Lee YS, Wentzel EA, Arking DE, West KM, Dang CV, Thomas-Tikhonenko A and Mendell JT: Widespread microRNA repression by Myc contributes to tumorigenesis. Nat Genet 40: 43-50, 2008. 